\title{
Renal function and metabolic syndrome components on cardiovascular and all-cause mortality
}

\author{
Kuo-Liong Chien ${ }^{\mathrm{a}, \mathrm{b}, *}$, Hsiu-Ching Hsu ${ }^{\mathrm{b}}$, Yuan-Teh Lee ${ }^{\mathrm{b}, \mathrm{c}}$, Ming-Fong Chen ${ }^{\mathrm{b}}$ \\ a Institute of Preventive Medicine, College of Public Health, National Taiwan University, Taipei, Taiwan \\ ${ }^{\mathrm{b}}$ Department of Internal Medicine, National Taiwan University Hospital, Taipei, Taiwan \\ ${ }^{\mathrm{c}}$ Ming-Shen Medical Center, Taoyuang, Taiwan
}

Received 15 December 2006; received in revised form 31 May 2007; accepted 31 July 2007

Available online 12 September 2007

\begin{abstract}
Objectives: Impaired renal function and metabolic syndrome have been associated with risk of cardiovascular disease (CVD). We investigated their roles in CVD and all-cause death among ethnic Chinese population.

Methods: We followed up a cohort of 11429 men and 7472 women aged 20 years and older for an average 4.9 years (median: 3.5 , inter-quartile range: $2.7-7.9$ ) from the tertiary hospital health check-up population.

Results: CVD death rates increased when the quintiles of each variable progressed. Metabolic syndrome was a significant predictor for CVD death, with relative risk of up to 4.68. In the multivariate adjusted model that included metabolic syndrome, quintiles of serum creatinine concentrations, estimated glomerular filtration rate (GFR), and uric acids were significantly associated CVD death, with the highest relative risk of creatinine concentration $(11.22,95 \%$ confidence interval $[\mathrm{CI}]: 2.43-51.7, P$ for trend: $<0.001)$. Serum creatinine concentrations and estimated GFR had the higher areas under ROC curves of CVD death $(0.76,95 \%$ CI: $0.71-0.80$ for creatinine and 0.76 , $95 \%$ CI: $0.72-0.81$ for estimated GFR). The two marker models showed that metabolic syndrome and impaired renal function had the most significant roles in predicting CVD deaths; the multivariate relative risk was 30.6 (95\% CI: 3.7-254, P: 0.002) in participants with the highest creatinine and presence of metabolic syndrome compared with those with the lowest and absence of metabolic syndrome.

Conclusions: Impaired renal function and metabolic syndrome are important risk factors for CVD and all-cause deaths among ethnic Chinese. (C) 2007 Elsevier Ireland Ltd. All rights reserved.
\end{abstract}

Keywords: Metabolic syndrome; Renal function; Cardiovascular death; Chinese

\section{Introduction}

Metabolic syndrome is considered an important risk factor for cardiovascular disease [1,2]. Impaired renal function was also reported to be associated with cardiovascular mortality in several studies, such as the general US population [3], in the elderly [4], and specific disease subjects, such as congestive heart failure patients [5]. There is an estimated 6.2 million people in the US with serum creatinine level higher than $1.5 \mathrm{mg} / \mathrm{dL}$. Chronic renal insufficiency is likewise associated with cardiovascular mortality [6]. Every $1 \mathrm{~mL} / \mathrm{min}$ decrease in creatinine clearance increased mortality by $1 \%$ [5].

\footnotetext{
* Corresponding author.

E-mail address: klchien@ha.mc.ntu.edu.tw (K.-L. Chien).
}

Cross-sectional studies have shown significant relationship between chronic kidney disease, metabolic syndrome and atherosclerotic risk among population $[7,8]$, but data on prospective cohort for further cardiovascular events were limited. In addition, the potential additive effects of the chronic kidney disease and metabolic syndrome, after considering the individual markers, were still unknown in ethnic Chinese population. Thus, this cohort study, based on the health examination of subjects in one tertiary hospital, provided an empirical observational dataset for evidence of biomarkers on cardiovascular and all-cause mortality. The focus was on renal function, uric acid, and metabolic syndrome factors, particularly the potential mediating effects among these risk factors on cardiovascular and all-cause mortality. 


\section{Methods}

\subsection{Study designs and study subjects}

This is a prospective cohort study design involving participants who undertook health examinations at the National Taiwan University Hospital in four different periods (1995, 1996, 2001, and 2002). Participants were recruited according to the criteria of age. Oral informed consents were obtained. The exclusion criteria included incomplete baseline available data, severely impaired renal function (serum creatinine levels $\geq 6 \mathrm{mg} / \mathrm{dL}$ ), and co-morbidities of cancer and cardiovascular disease (CVD) including history of coronary heart disease and stroke. We included a total of 11429 men and 7472 women who were $\geq 20$ years and completed questionnaire in the baseline.

All of the subjects received the standard questionnaire and physical examination in the Health Management Center, National Taiwan University Hospital. The protocol was approved by the IRB of National Taiwan University. Structured questionnaires on family history, lifestyle habits, and medical history were collected, as well as anthropometric measurements, such as body weight, body height and waist circumference.

Physical examination and 12-lead electrocardiogram were likewise performed. Blood pressure was measured in a resting position while body mass index (BMI) was calculated as weight (in $\mathrm{kg}$ )/height (in $\mathrm{m})^{2}$. Blood pressure was measured twice in the right arm by a mercury sphygmomanometer with the subject seated comfortably and arms supported and positioned at the level of the heart after $10 \mathrm{~min}$ rest.

\subsection{Laboratory measurements}

Procedures for blood sampling and analytic methods were as previously described [9]. In brief, blood sample was collected from each participant after at least a $12 \mathrm{~h}$ fasting. Serum total cholesterol levels were measured using the CHOD-PAP method (Boehringer Mannheim, Germany) while HDL-C was measured following precipitation of apolipoprotein B-containing lipoproteins, with phosphotungstic acid and magnesium ions (Boehringer Mannheim, Germany)[10]. Triglyceride concentrations were measured by the GPO-DAOS method (Wako Co., Japan). All of the biochemical measurements, including the aforementioned lipids, uric acid, and creatinine concentrations, were measured using a Hitachi 7450 automated analyzer (Hitachi, Japan). All of the sample measurements were carried out in a single hospital. The coefficients of variation of above measurements were around $5 \%$.

Two renal function indices were used: serum creatinine levels and estimated glomerular filtration rate (GFR) ( $\mathrm{mL} / \mathrm{min})$, which was calculated by the Cockcroft-Gault equations (i.e. $(140$-age $) \times($ weight in $\mathrm{kg}) /(72 \times$ creatinine in $\mathrm{mg} / \mathrm{dL}$ ) for men, and the value multiplied by 0.85 for women due to smaller muscle mass [6]. We did not use other indices of renal functions, cystatin-C, because the data was not available. Another index, Modification of Diet in Renal Disease (MDRD) also provided a similar renal function index as Cockcroft-Gault equation [11]. However, MDRD estimated GFR was reported to underestimate the GFR systematically among patients without chronic kidney disease [12], so we adopted Cockcroft-Gault estimated GFR as renal function indicator in the study. Metabolic syndrome status was defined according to the criteria set in the Third Adult Treatment Panel of the National Cholesterol Education Program [13], modified for use with Asian waist circumference cut points [14]. Therefore, three of the following five criteria were grounds for definition: (1) blood pressure of at least $130 / 85 \mathrm{mmHg}$ or treatment for hypertension; (2) serum triglyceride of at least $150 \mathrm{mg} / \mathrm{dL}$; (3) HDL-cholesterol $<40 \mathrm{mg} / \mathrm{dL}$ in men and $<50 \mathrm{mg} / \mathrm{dL}$ in women; (4) fasting glucose of $110 \mathrm{mg} / \mathrm{dL}$ or more; and (5) waist circumference $\geq 90 \mathrm{~cm}$ in men, $\geq 80 \mathrm{~cm}$ in women or BMI of $27 \mathrm{~kg} / \mathrm{m}^{2}$ or greater. The metabolic syndrome components were categorized into quintiles to evaluate the additive risks on the endpoints.

\subsection{Endpoint ascertainment}

Information on mortality and causes of death were obtained by linking the identification numbers of the study subjects to a national databank provided by the National Health Administration, which was updated to the end of 2005. We defined CVD mortality by the death codes 390-459 from the 9th version of the International Classification of Diseases (ICD).

\subsection{Statistical analysis}

The data were presented with mean, standard deviation and contingency tables, specified by serum creatinine quintiles. We tested the all-cause mortality rates in the study population and compared with the Taiwanese 2004 census data. We specified the mortality rates by metabolic syndrome numbers, quintiles of renal function indices, and uric acid concentrations. The two endpoints, cardiovascular death mortality and total-cause mortality rates were estimated in the quintiles.

Because a linear relationship between risk factors and the two endpoints was not assumed, we stratified serum creatinine, estimated GFR, and uric acid into quintiles. We tested the relative risk (RR) and $95 \%$ confidence interval (CI) of the four quintile groups and compared these with the lowest quintile as baseline. We grouped metabolic syndrome numbers into five groups, i.e., 0, 1, 2, 3, and 4-5, and treated with dummy variables to estimate the potential non-linear relationship. The Cox proportional hazards model was used to estimate the model.

To test for linear trend across creatinine, estimated GFR and uric acid categories, we used the median levels within quintiles as a continuous variable. We used univariate (four 
Table 1

Basic characteristics of the study subjects according to serum creatinine quintiles

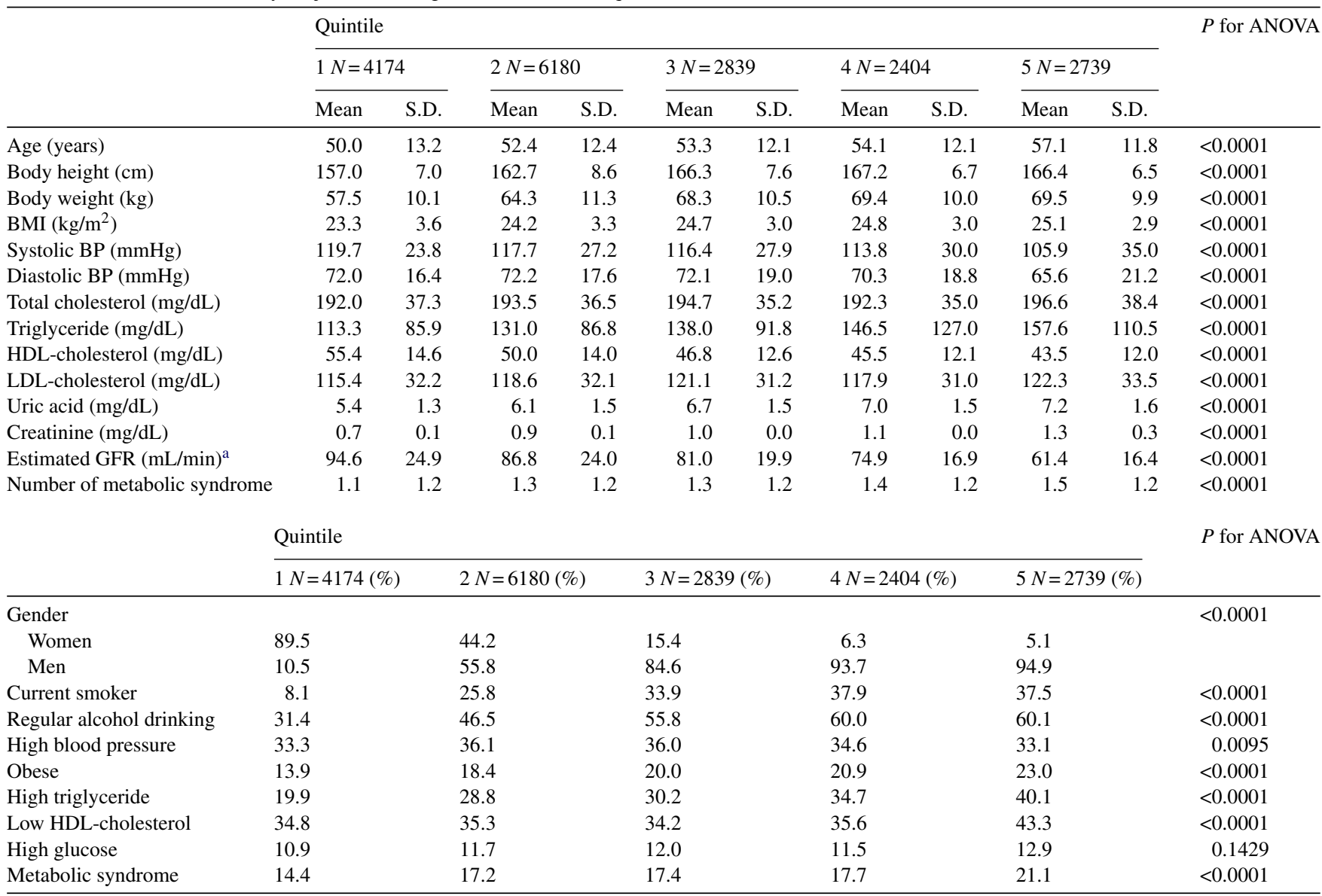

Abbreviations: BMI, body mass index; BP, blood pressure; GFR, glomerular filtration rate; HDL, high density lipoprotein; LDL, low density lipoprotein; S.D., standard deviation.

a Calculated by the Cockcroft-Gault equation.

dummy variables) and multivariate adjusted models to evaluate the relative risks and $95 \%$ confidence intervals, stratified by metabolic syndrome status. We adjusted for multiple confounding factors as follows: entry periods $(1995,1996,2001$, and 2002 cohort), age (20-39, 40-49, 50-59, 60-69, and $\geq 70$ years old), body mass index $(<18,18-19.9,20-21.9$, 22-23.9, 24-25.9, 26-27.9 and $\geq 28 \mathrm{~kg} / \mathrm{m}^{2}$ ), smoking (yes/no), alcohol drinking habits (regular/no), and baseline metabolic syndrome components such as high blood pressure, high triglyceride, low HDL-C, obesity, and high glucose level.

We tested the hypotheses by the following strategies. First, we estimated the hazard risk (HR) of top versus bottom quintiles, and examined the significance levels of test of trend. Second, we used the area under the curve of receiver operative characteristics (ROC curve) to compare the discriminative ability of various risk factors [15]. The area under ROC curve was considered as a global performance indicator for a prognostic factor. Third, likelihood ratio test statistics were used to compare the model fitting. We compared models with and without the four dummy variables and calculated
2Ln(likelihood) values to test the difference between two models. Finally, we used two marker models, one for creatinine quintiles and another for various metabolic syndrome components or uric acid quintiles, with adjustments for age, gender, body mass index, and metabolic syndrome number covariates. This two-marker model provided the evidence of the additive effects when the original model included the creatinine quintiles. We tested for trends with median values in every quintile and presented the relative risks of top versus bottom quintiles of the two markers. We also compared the likelihood ratio test values for these two markers to evaluate the significance levels of the additive markers.

We further tested for possible effect modification of creatinine, estimated GFR or uric acid levels with metabolic syndrome status by stratification and plotted the multivariate relative risks, adjusting for the above confounding factors, and examined the risks compared with baseline category. All analyses were performed with SAS version 9.1 (SAS Institute, Cary, NC) and Stata version 9.1 (Stata Corporation, College Station, Texas). A two-sided $P<0.05$ was considered statistically significant. 
Table 2

Incidence rates, relative risks and 95\% confidence intervals of CVD deaths during follow-up, according to metabolic syndrome numbers, renal function quintiles, and uric acid levels in the study population

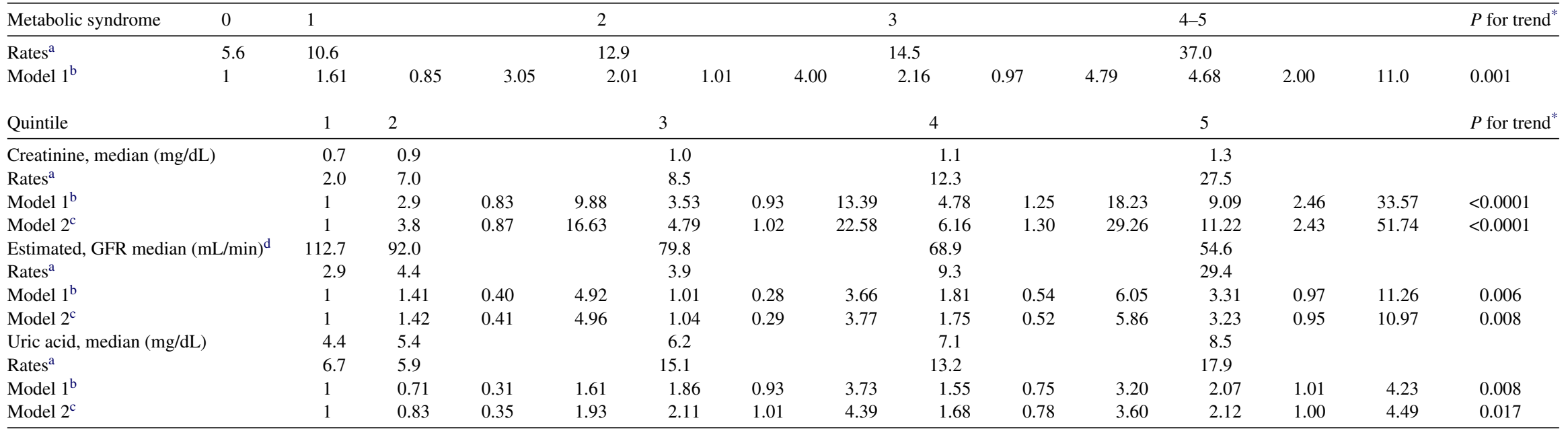

Abbreviation: CVD, cardiovascular death.

a Rates per 10,000 person-years.

${ }^{\mathrm{b}}$ Model 1: adjusted for entering period (four categories), age groups (five categories), gender, body mass index (seven categories), alcohol intake and smoking status.

c Model 2: more four dummy variables of metabolic syndrome components in additional to Model 1.

${ }^{\mathrm{d}}$ Calculated by the Cockcroft-Gault equation, reverse order.

* $P$ test for trend based on median levels in each quintile used as a continuous variable. 


\section{Results}

The basic characteristics of the study participants, including anthropometric, blood pressure, lipid profiles, uric acid, creatinine, and estimated GFR were presented in Table 1, according to quintiles of serum creatinine concentrations. Participants with the highest creatinine quintile are likely to have older age, higher body height, weight, body mass index, total and LDL-cholesterol, triglyceride, uric acid and metabolic syndrome numbers, yet to have lower blood pressure, HDL-cholesterol, and estimated GFR, compared with those with the lower creatinine quintiles (Table 1). Participants with higher creatinine concentrations are more likely to be male obese smoker, to have regular exercise, and to have more metabolic syndrome components than those with lower creatinine levels.

The age and gender adjusted Spearman correlation coefficients between various metabolic syndrome and renal function indices were presented in Supplementary Table 1. Renal function indices, such as serum creatinine, estimated GFR, and uric acid were mildly associated with metabolic syndrome and its components (coefficients $\approx 0.1-0.3$ ), implying that renal function indices were independent of atherosclerotic risk factors and suitable for markers to detect the interaction effects of renal function and atherosclerotic risk factors.

After an average of 4.9 years (median: 3.5, inter-quartile range: 2.7-7.9) of follow-up, 500 subjects died, including 106 due to cardiovascular deaths. The all-cause death rates in the study population were 54.5 per 10,000 person-year (6.38 for men, 3.98 for women), and were not significantly different from the total-cause death rates in the 2004 Taiwan data (59.0 per 10,000 person-year, binomial test, $P=0.074)$. Table 2 shows the quintile-specific median values, CVD mortality rates, and multivariate adjusted relative risk and its 95\% confidence interval (CI) during the follow-up periods across metabolic syndrome numbers, renal func-

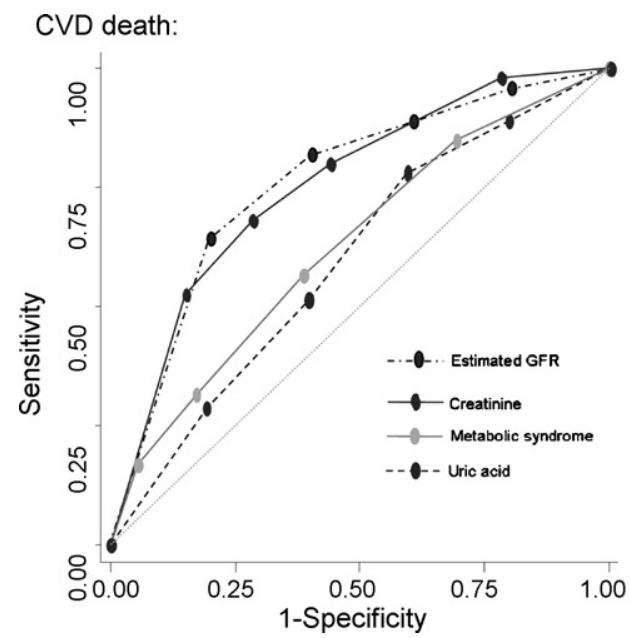

tion indices, and uric acid quintiles in the baseline. CVD death rates increased when the quintiles of each variable progressed. Metabolic syndrome was a significant predictor for CVD deaths, with relative risk up to 4.68 in the fifth quintile. Among the multivariate adjusted models that included metabolic syndrome, quintiles of serum creatinine concentrations, estimated GFR, and uric acids were significantly associated CVD death, with the highest relative risk of creatinine concentration (11.22). The all-cause death rate pattern was similar to the CVD rates (Supplementary Table 2) except for uric acid, which did not elicit statistically significant trend for predicting all-cause death (test for trend, $P=0.32$ ). We also examined the interaction effects of genders and various variables according to the likelihood ratio tests between the two nested models, and we found nonsignificant interaction between genders in most variables (data not shown).

Fig. 1 shows the ROC curves of CVD death and all-cause death events by various quintiles of risk factors. The estimated areas under ROC curves for predicting CVD and all-cause deaths were listed in Table 3. We found that serum creatinine concentrations and estimated GFR had the higher areas under ROC curves for the two endpoints, and different from the uric acid quintiles.

We detected the synergistic effects of metabolic syndrome and serum creatinine concentrations for predicting CVD and all-cause deaths in the study population (Fig. 2). Metabolic syndrome was associated with the endpoints, and creatinine significantly increased the risks. The statistics of test of homogeneity for metabolic syndrome status were not significant.

We used two marker models to detect the possible additive risks of another marker into CVD and all-cause death rates (Supplementary Table 3). When the model included creatinine concentrations, only low HDL and high LDL quintiles added significant likelihood ratio test statistics to predict CVD death $(P<0.05)$.

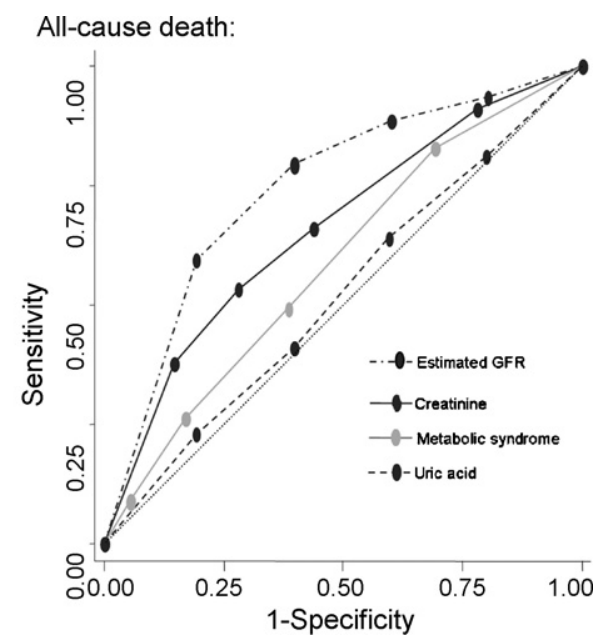

Fig. 1. The ROC curves for CVD death (left) and all-cause death (right) according to quintiles of different renal function indices, metabolic syndrome numbers, and uric acid quintiles in the study population. 
Table 3

Area under ROC curves of various markers for predicting CVD death and all-cause death in the study population

\begin{tabular}{|c|c|c|c|c|c|c|c|c|}
\hline & \multicolumn{3}{|c|}{ CVD death* } & & \multicolumn{3}{|c|}{ All-cause death* } & \\
\hline & Area & S.E. & $95 \% \mathrm{CI}$ & & Area & S.E. & $95 \% \mathrm{CI}$ & \\
\hline Estimated GFR quintiles $^{\mathrm{a}}$ & 0.762 & 0.023 & 0.718 & 0.806 & 0.746 & 0.011 & 0.724 & 0.767 \\
\hline Creatinine quintiles & 0.756 & 0.022 & 0.712 & 0.799 & 0.661 & 0.013 & 0.637 & 0.686 \\
\hline Metabolic syndrome numbers & 0.628 & 0.027 & 0.575 & 0.682 & 0.589 & 0.012 & 0.565 & 0.613 \\
\hline Uric acid quintiles & 0.598 & 0.026 & 0.547 & 0.650 & 0.521 & 0.013 & 0.495 & 0.547 \\
\hline
\end{tabular}

Abbreviations: CVD, cardiovascular death; CI: confidence interval; GFR, glomerular filtration rate; S.E., standard error.

a Calculated by the Cockcroft-Gault equation.

* Difference among four areas: $P<0.001$.
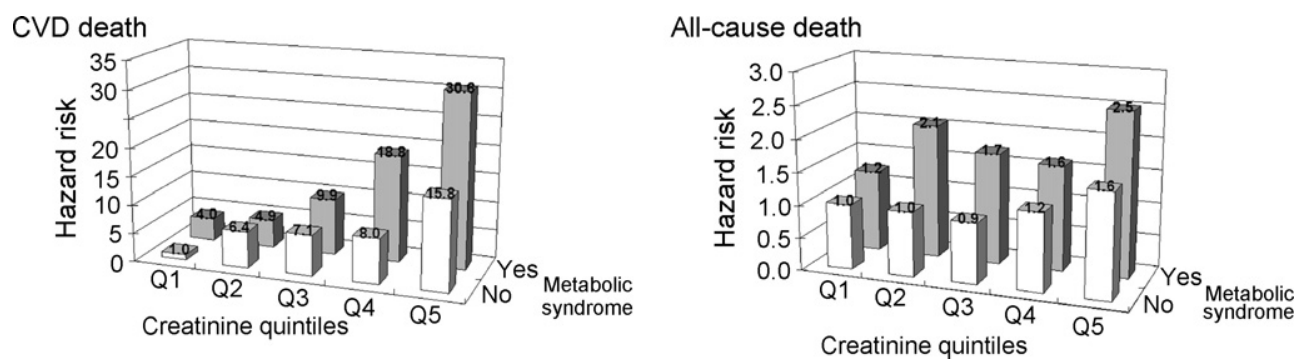

Fig. 2. Relative risks of CVD death (left) and all-cause death (right) according to the metabolic syndrome status and creatinine quintiles in the study population.

\section{Discussion}

Renal function indices were strongly associated with CVD and all-cause death rates in the ethnic Chinese who undertook health examination in one tertiary hospital. We clearly demonstrated the additive risks of impaired renal function, uric acid and metabolic syndrome components of CVD death risk. Our study not only demonstrated the magnitude of influence of renal function but also showed the potential risks of various metabolic syndrome and lipid profiles on CVD and all-cause death.

Researchers argued that could there be a causal relationship between chronic renal insufficiency and CVD death, or just is renal insufficiency only a marker for co-morbidities or disease severity [16]. Evidence on clustering of renal insufficiency, metabolic syndrome and atherosclerotic risk factors from observational studies implied renal function was associated with metabolic syndrome components $[7,8]$. There are several evidences that support the role of renal impairment in CVD risk, as well as the potential mechanisms for cardio-renal interaction [17]. First, impaired renal function worsens hypertension, activates the renin-angiotensin system, increases sodium and water retention, and induces hyper-coagulation and inflammatory reactions in the body, which aggravates atherosclerotic burden [16]. Second, excess co-morbidities of metabolic syndrome components also contribute to worsening CVD risks among subjects with chronic or end-stage renal disease. Finally, chronic renal disease has multiple effects on vascular dysfunctions, which induce atherosclerosis. Our data showed that substantial effects of renal impairment on CVD events, and the results were consistent with other ethnic population-based cohorts, such as US-based [3] and Asian based studies [18]. Nation-wide sur- vey showed high prevalence of renal impairment but low awareness in Taiwanese general population [19]; therefore, screening on renal function is mandatory for primary prevention of CVD in general population.

Previous studies have shown that metabolic syndrome and its components were important risk factors for CVD death [20]. The existence of metabolic syndrome enforced the effects of renal insufficiency on CVD and all-cause death. The interrelationships among obesity, metabolic syndrome, and chronic kidney disease would worsen further CVD events among patients with renal insufficiency $[7,8,17]$. We used traditional NCEP definition of metabolic syndrome, and the other definitions, such as IDF (International Diabetes Federation) and AHA (American Heart Association) have similar prognostic implications for the outcomes. Also, prior studies on Asian populations have demonstrated that, while BMI and metabolic syndrome are important predictors of CVD and death, substantially lower values of BMI (even those considered in the "normal" range for US-based populations) are still predictive of increasing poor prognosis. Our data also showed mild obesity still had high risks for CVD mortality.

There were inconsistent study results on the relationship between hyperuricemia and CVD risk $[21,22]$. Our previous study results, based on a community-based cohort, have demonstrated that uric acid had marginally statistically significant roles for CVD events [23]. Our present data showed that uric acid role as CVD death remained to be significant after multivariate and metabolic syndrome adjustments. Metabolic syndrome induces high oxidative stress and the accompanying hyperuricemia worsens the oxidative stress [24]. Furthermore, uric acid stimulates vascular smooth muscle proliferation and induces endothelial dysfunction [25]. The increase of uric acid decreases endothelial nitric oxide 
production, and consequently, makes peripheral tissue resistant to insulin effects and results in endothelial dysfunction [26]. Moreover, high uric acid is associated with increased renal glomerular pressure and increased renal sodium reabsorption, and these renal reactions are greatly enhanced by high insulin concentrations [27]. In addition, hyperuricemia was associated with insulin resistance markers, including triglycerides, microalbuminuria and impaired glucose tolerance [25]. The combination effects of insulin resistance and hyperuricemia on renal functions resulted in high cardiovascular risk.

Our results have several clinical implications. First, mild renal function impairment should be an indicator for clinical monitoring and close follow-up of renal function, such as serum creatinine concentrations or estimated GFR in the general population is mandatory. Second, metabolic syndrome and renal function makers had additive risks on CVD and all-cause death. Hence, lifestyle intervention on metabolic syndrome prevention and salt restriction on renal function should be emphasized in the primary care setting and in public health. Further randomized controlled trial designs can answer the effects of prevention of metabolic syndrome and renal function deterioration on clinical outcomes.

On the other hand, this study had several limitations. First, only one baseline measurement of renal functions and metabolic syndrome indices was used and repeated measurements in the model were not incorporated. There were potential non-differential misclassification errors in the exposure and outcome ascertainment. The actual risks should be more significant because of the toward-to-null trend in non-differential misclassification. Second, the study subjects were recruited from one health examination cohort, which is not representative of the general ethnic Chinese population. Individuals who undertook the health check-up had higher education levels, were more apt to health behavior and medical care, and had higher socioeconomic status and motivation for health promotion. Nonetheless, the results were valid in the observational cohort design in the causal inference. Third, the limited numbers of CVD and all-cause deaths might have less power to detect the significance levels of risks of the risk factors, especially since there was no stratification of events into subgroups. Finally, the validity and reliability of using ICD-9 codes to define CVD mortality is limited.

In conclusion, renal functions and metabolic syndrome had synergistic effects on CVD and all-cause death rates among ethnic Chinese. Optimal treatments on renal function preservation and prevention of metabolic syndrome are mandatory for the prevention of CVD and all-cause deaths.

\section{Acknowledgements}

The authors wish to thank the staff of the Heath Management Center, National Taiwan University Hospital, for their assistance in the study. The authors also express their grati- tude to the participants in the study. This study was supported in part by the National Science Council, Taiwan.

\section{Appendix A. Supplementary data}

Supplementary data associated with this article can be found, in the online version, at doi:10.1016/j.atherosclerosis. 2007.07.037.

\section{References}

[1] Grundy SM. Point: the metabolic syndrome still lives. Clin Chem 2005;51:1352-4.

[2] McNeill AM, Rosamond WD, Girman CJ, et al. The metabolic syndrome and 11-year risk of incident cardiovascular disease in the atherosclerosis risk in communities study. Diabetes Care 2005;28: 385-90.

[3] Muntner P, He J, Hamm L, Loria C, Whelton PK. Renal insufficiency and subsequent death resulting from cardiovascular disease in the United States. J Am Soc Nephrol 2002;13:745-53.

[4] Fried LF, Shlipak MG, Crump C, et al. Renal insufficiency as a predictor of cardiovascular outcomes and mortality in elderly individuals. J Am Coll Cardiol 2003;41:1364-72.

[5] McAlister FA, Ezekowitz J, Tonelli M, Armstrong PW. Renal insufficiency and heart failure: prognostic and therapeutic implications from a prospective cohort study 2. Circulation 2004;109:1004-9.

[6] K/DOQI clinical practice guidelines for chronic kidney disease: evaluation, classification, and stratification. Am J Kidney Dis 2002;39: S1-266.

[7] Kurella M, Lo JC, Chertow GM. Metabolic syndrome and the risk for chronic kidney disease among nondiabetic adults. J Am Soc Nephrol 2005;16:2134-40.

[8] Gomez P, Ruilope LM, Barrios V, et al. Prevalence of renal insufficiency in individuals with hypertension and obesity/overweight: the FATH study. J Am Soc Nephrol 2006;17:S194-200.

[9] Chien KL, Yang CY, Lee YT. Major gene effects in systolic and diastolic blood pressure in the families receiving health examination in Taiwan. J Hypertens 2003;21:1-7.

[10] Lopes-Virella M, Stone P, Ellis S, Colwell JA. Cholesterol determination in high-density lipoproteins separated by three different methods. Clin Chem 1977;23:882-4.

[11] de Jong PE, Gansevoort RT. Screening techniques for detecting chronic kidney disease. Curr Opin Nephrol Hypertens 2005;14: 567-72.

[12] Rule AD, Larson TS, Bergstralh EJ, et al. Using serum creatinine to estimate glomerular filtration rate: accuracy in good health and in chronic kidney disease. Ann Intern Med 2004;141:929-37.

[13] Executive Summary of The Third Report of The National Cholesterol Education Program (NCEP) Expert Panel on Detection, Evaluation, And Treatment of High Blood Cholesterol In Adults (Adult Treatment Panel III). JAMA 2001;285:2486-97.

[14] Tan CE, Ma S, Wai D, Chew SK, Tai ES. Can we apply the National Cholesterol Education Program Adult Treatment Panel definition of the metabolic syndrome to Asians? Diabetes Care 2004;27: 1182-6.

[15] Hanley JA, McNeil BJ. The meaning and use of the area under a receiver operating characteristic (ROC) curve. Radiology 1982;143: 29-36.

[16] McCullough PA. Why is chronic kidney disease the "spoiler" for cardiovascular outcomes? J Am Coll Cardiol 2003;41:725-8.

[17] Lastra G, Manrique C, Sowers JR. Obesity, cardiometabolic syndrome, and chronic kidney disease: the weight of the evidence. Adv Chronic Kidney Dis 2006;13:365-73. 
[18] Irie F, Iso H, Sairenchi T, et al. The relationships of proteinuria, serum creatinine, glomerular filtration rate with cardiovascular disease mortality in Japanese general population. Kidney Int 2006;69:1264-71.

[19] Hsu CC, Hwang SJ, Wen CP, et al. High prevalence and low awareness of CKD in Taiwan: a study on the relationship between serum creatinine and awareness from a nationally representative survey. Am J Kidney Dis 2006;48:727-38.

[20] Kahn R, Buse J, Ferrannini E, Stern M. The metabolic syndrome: time for a critical appraisal: joint statement from the American Diabetes Association and the European Association for the Study of Diabetes. Diabetes Care 2005;28:2289-304.

[21] Nakanishi N, Okamoto M, Yoshida H, et al. Serum uric acid and risk for development of hypertension and impaired fasting glucose or Type II diabetes in Japanese male office workers. Eur J Epidemiol 2003;18:523-30.

[22] Taniguchi Y, Hayashi T, Tsumura K, et al. Serum uric acid and the risk for hypertension and Type 2 diabetes in Japanese men: The Osaka Health Survey. J Hypertens 2001;19:1209-15.
[23] Chien KL, Hsu HC, Sung FC, et al. Hyperuricemia as a risk factor on cardiovascular events in Taiwan: The Chin-Shan Community Cardiovascular Cohort Study. Atherosclerosis 2005;183:14755.

[24] Hansel B, Giral P, Nobecourt E, et al. Metabolic syndrome is associated with elevated oxidative stress and dysfunctional dense high-density lipoprotein particles displaying impaired antioxidative activity. J Clin Endocrinol Metab 2004;89:4963-71.

[25] Johnson RJ, Kang DH, Feig D, et al. Is there a pathogenetic role for uric acid in hypertension and cardiovascular and renal disease? Hypertension 2003;41:1183-90.

[26] Feig DI, Mazzali M, Kang DH, et al. Serum uric acid: a risk factor and a target for treatment? J Am Soc Nephrol 2006;17:S6973.

[27] Quinones Galvan A, Natali A, Baldi S, et al. Effect of insulin on uric acid excretion in humans. Am J Physiol 1995;268:E1-5. 